\title{
ORIGINAL
}

\section{DISEÑO Y VALIDACIÓN DE UN CUESTIONARIO PARA EVALUAR LA SATISFACCIÓN DE LOS PACIENTES ATENDIDOS EN LAS CONSULTAS EXTERNAS DE UN HOSPITAL DE MADRID EN 2006}

\author{
Susana Granado de la Orden, Cristina Rodríguez Rieiro, María del Carmen Olmedo Lucerón, \\ Ana Chacón García, Dolores Vigil Escribano y Paz Rodríguez Pérez \\ Servicio de Medicina Preventiva y Gestión de Calidad. Hospital General Universitario Gregorio Marañón. Madrid.
}

\section{RESUMEN}

Fundamento: Para conocer la calidad de la asistencia sanitaria es imprescindible incorporar el punto de vista del paciente realizando estudios de satisfacción. Las encuestas de satisfacción precisan unas propiedades psicométricas que garanticen su fiabilidad y validez. El objetivo de este trabajo es diseñar y validar un cuestionario para conocer la satisfacción de los pacientes con las consultas externas hospitalarias.

Métodos: Estudio de validación de un cuestionario autocumplimentado, diseñado por un grupo de expertos, compuesto por 12 preguntas que se aplicó a una muestra de 677 pacientes de las consultas externas del hospital Gregorio Marañón. Se analizó el comportamiento métrico del cuestionario en cuanto a validez de constructo, de criterio y capacidad predictiva y la consistencia interna.

Resultados: Se analizó el comportamiento métrico del cuestionario respecto a la validez de constructo identificándose 2 factores "calidad clínica" y "calidad administrativa" que explican el $63 \%$ de la varianza, validez de criterio concurrente (coeficiente de correlación de Spearman de 0,85 respecto al criterio de referencia "satisfacción global con la atención recibida en consultas externas", OR 1,19 respecto al criterio de referencia "conoce el nombre del médico que le atiende en la consulta"), capacidad predictiva del cuestionario medida mediante análisis discriminante obteniéndose un $84 \%$ de los casos clasificados correctamente y consistencia interna (Alfa de Cronbach 0,90).

Conclusiones: El cuestionario reúne suficientes propiedades psicométricas como para que pueda ser considerado una herramienta útil y fiable para medir la satisfacción de los pacientes con las consultas externas hospitalarias.

Palabras clave: Satisfacción del paciente. consultas externas hospitalarias, calidad de la atención de salud, cuestionario, reproducibilidad de los resultados.

Correspondencia:

Susana Granado de la Orden

Hospital General Universitario Gregorio Marañón

C/ Ibiza, 2. 28071 Madrid

Correo electrónico: sgranado.hgugm@salud.madrid.org

\section{ABSTRACT}

\section{Design and Validation of a Questionnaire to Evaluate Satisfaction of Patients of the Hospital Outpatient Clinics in Madrid, Spain, 2006}

Background: In order to improve the quality of health care it is necessary to know the point of view of the patients through satisfaction surveys. Satisfaction surveys need some psychometric properties to guarantee its reliability and validity. The aim of this paper is to design and to validate a questionnaire to determine the satisfaction of patients of the hospital outpatient clinics.

Methods: Validation of a satisfaction questionnaire, designed by an expert group and composed for 12 questions. The questionnaire was given to a sample of 677 patients attended in the hospital Gregorio Marañón outpatient clinics. The psychometric properties of the questionnaire were established: construct validity, concurrent criterion validity, predictive capacity and internal consistency.

Results: Two dimensions were identified in the construct validity analyses, explaining $63 \%$ of the variance: "clinical quality" and "administrative quality". The concurrent validity analysis showed a Spearman's rank correlation coefficient of 0.85 related to the standard criterion "global satisfaction with the attention provided in outpatient clinics" and an OR of 1.19 related to the criterion "do you know the name of the physician who attended you?" The predictive capacity of the questionnaire was measured by discriminated analysis, obtaining $84 \%$ of correct classified, and the internal consistency test gave a Cronbach's alpha of 0.90 .

Conclusions: Our questionnaire has enough psychometric properties to be considered an useful and feasible tool to measure the satisfaction of patients of the hospital outpatient clinics.

Key words: Patient satisfaction. Outpatient clinics, hospital. Quality control. Questionnaires. Reproducibility of results. 


\section{INTRODUCCIÓN}

Desde un punto de vista cercano a la gestión la calidad se puede definir como "el conjunto de propiedades y características de un producto o servicio que le confieren su aptitud para satisfacer unas necesidades expresadas o implícitas"1. Esto implica detectar las necesidades, tener un modelo de referencia para comparar, y conseguir satisfacer dichas necesidades y expectativas del cliente. Actualmente, la valoración de la calidad de los servicios ofertados y el desarrollo posterior de las actividades de mejora constituyen un proceso inseparable del ejercicio profesional ${ }^{2,3}$. El papel que juega la opinión de los usuarios, sus percepciones y los juicios que realizan sobre los servicios recibidos son aspectos claves en la definición de la calidad y su valoración es imprescindible para prestar una correcta asistencia sanitaria ${ }^{4,5}$. La percepción del paciente es, por tanto, una de las medidas principales a la hora de evaluar y mejorar la calidad de la asistencia ${ }^{6,7}$. El objetivo final será detectar la presencia de problemas en la asistencia y establecer propuestas para mejorar la atención a través de la valoración que el paciente manifieste del servicio recibido $^{8}$.

Existen diferentes métodos que nos permiten aproximarnos al conocimiento del nivel de satisfacción del usuario con el sistema sanitario, como el análisis de quejas y sugerencias $^{9}$ o la realización de estudios empleando metodología cualitativa ${ }^{10}$. Sin embargo, en nuestro medio, los estudios de satisfacción con los servicios sanitarios se realizan, habitualmente, mediante técnicas de investigación cuantitativa: cuestionarios o encuestas de satisfacción ${ }^{11,12}$.

Las encuestas de satisfacción de pacientes requieren una serie de propiedades psicométricas que garanticen su fiabilidad y validez, especialmente cuando el propósito es extrapolar los resultados obtenidos a la población ${ }^{13}$. De ahí la creciente necesidad de realizar encuestas de satisfacción validadas en nuestro medio, asegurando que el cuestionario elaborado y los ítems elegidos sean adecuados para medir lo que se pretende medir (validez de contenido), y que los componentes o dimensiones del cuestionario estén claramente definidos de forma que cada uno contribuya al total de la escala de forma independiente (validez de constructo ${ }^{14}$. De igual forma, las medidas obtenidas con el cuestionario deben asemejarse o correlacionarse con las obtenidas con un método Gold Standard (validez de criterio) y el cuestionario debe ser fiable (valorado a través de la consistencia interna, la estabilidad temporal y la concordancia interobservadores) y preciso, es decir, capaz de ofrecer en su empleo repetido resultados veraces y constantes en condiciones similares de utilización. Por último, el cuestionario debe de ser sensible al cambio, es decir, capaz de detectar diferencias en la magnitud del constructo, además de ser aceptado por todos los agentes implicados (entrevistador, entrevistado, investigador, etc.).Validar un cuestionario consiste en comprobar, ratificar o verificar que cumple estas propiedades de validez y fiabilidad, mediante la realización de aplicaciones repetidas y mediciones contrastadas.

El gran volumen de pacientes atendido en las consultas, sus expectativas ${ }^{15}$ y las características propias de la atención en consultas externas (masificación, largas listas de espera, citas sucesivas, etc.) hacen conveniente estudiar la satisfacción con las consultas externas hospitalarias mediante un cuestionario diseñado específicamente para estos pacientes. Sin embargo, existen pocos estudios realizados en nuestro medio para conocer la satisfacción de los pacientes atendidos en consultas externas ${ }^{16-19}$, y la mayoría de ellos han sido realizados para consultas específicas utilizando cuestionarios no validados ${ }^{20}$.

El objetivo principal de este trabajo es diseñar y valorar la validez y fiabilidad de 
un cuestionario para conocer la satisfacción de los pacientes con el servicio prestado en las consultas externas hospitalarias.

\section{SUJETOS Y MÉTODOS}

La población de estudio fueron los pacientes atendidos en el "Pabellón de Consultas Externas" del Hospital General Universitario Gregorio Marañón de Madrid (HGUGM) donde se atienden el 50\% de las 470.000 consultas/año que se generan en el hospital. Se excluyó a los pacientes atendidos en la unidad de extracciones, y a los atendidos en las consultas de pruebas diagnósticas, debido a que las características de la atención en estas consultas difieren ampliamente del resto. Se calculó un tamaño muestral de 677 pacientes considerando un porcentaje esperado de satisfacción del $80 \%$, una precisión del $3 \%$ y un nivel de confianza del $95 \%$. El estudio fue realizado durante la primera semana del mes de junio de 2006.

\section{Construcción del cuestionario}

El cuestionario fue diseñado por un grupo de expertos en el que participó un equipo multidisciplinar de 7 miembros, formado por profesionales médicos y de enfermería de las consultas externas del hospital, del Servicio de Atención al Paciente, y del Servicio de Medicina Preventiva y Gestión de Calidad, y se contó con la colaboración de los expertos en estudios sociales de la Dirección General de Calidad de los Servicios y Atención al Ciudadano de la Comunidad de Madrid. Las dimensiones que a priori se pretendían explorar eran la accesibilidad, la relación interpersonal profesional sanitario-paciente y la comodidad del entorno. Los miembros del grupo elaboraron un listado de aspectos a evaluar a partir de su experiencia, al tiempo que se realizó una revisión bibliográfica en las bases de datos Medline y Embase utilizando los términos Mesh "patient satisfac- tion" y "outpatient clinics hospital" unidos mediante el operador "AND", para identificar aquellos aspectos que más influyen en la satisfacción con la atención en las consultas, prestando especial interés a los estudios cualitativos realizados con pacientes para conocer los aspectos que generan satisfacción/ insatisfacción ${ }^{21-24}$.

Se obtuvo un listado de 15 preguntas a partir del cual se diseñó el cuestionario final SUCE (Satisfacción de los Usuarios de las Consultas Externas) de 12 ítems que exploran aspectos relacionados con la satisfacción, cada uno de los cuales puntuaba en una escala ordinal desde 1 hasta 10 , siendo 1 la respuesta peor valorada y 10 la mejor. Junto a ellos se añadieron 12 preguntas sociodemográficas y de utilización de las consultas y 2 preguntas para ser utilizadas como criterios de referencia ("satisfacción global con la atención recibidas en consultas externas", con una escala de respuesta ordinal desde 1 hasta 10, y "conoce el nombre del médico que le atiende en consulta") y una pregunta abierta solicitando sugerencias. El cuestionario comprendía todos los aspectos de la atención, desde que el paciente solicita la citación para la consulta hasta que acude y es atendido. Antes del inicio del estudio se llevó a cabo un estudio piloto sobre una muestra aleatoria de 30 pacientes de las consultas externas para evaluar el grado de comprensibilidad de las preguntas, si la escala de respuesta era adecuada, si existía rechazo a contestar alguna de las preguntas y si el tiempo que era necesario invertir para responder a la encuesta era aceptable para el entrevistado. El estudio piloto conllevó la realización de algunos cambio menores en la formulación de las preguntas elaborándose la versión final del cuestionario.

La encuesta fue administrada durante el mes de junio de 2006 por los profesionales del Servicio de Medicina Preventiva y Gestión de Calidad junto con personal voluntario del Servicio de Atención al Paciente, que 
habitualmente colabora en la información y orientación a los pacientes que acuden a las consultas externas. La encuesta se entregaba a la entrada de las consultas, solicitando al paciente su colaboración con el objetivo de mejorar la atención y el paciente la depositaba en una urna a la salida. La encuesta era contestada por el propio paciente y en el caso de que hubiera algún tipo de dificultad o limitación y deseara cumplimentarla se le prestaba la ayuda necesaria. Las encuestas fueron recogidas diariamente y los datos fueron introducidos en una base de datos especialmente diseñada para el estudio con el programa informático ACCESS.

Análisis estadístico. Análisis descriptivo de las variables cuantitativas, comparando los valores medios mediante las pruebas $\mathrm{T}$ de Student y Análisis de la varianza (ANOVA) y Chi cuadrado para la comparación de proporciones.

Análisis de la validez del cuestionario. La validez de constructo se evaluó mediante la técnica del Análisis Factorial Exploratorio de Componentes Principales ${ }^{25}$, seguido de rotación Varimax para conseguir una mejor comprensión de la matriz resultante. Se comprobaron los supuestos de aplicación de la misma mediante la medida de adecuación muestral de Kaiser-Meyer-Olkin (KMO), el Test de esfericidad de Barlett y el determinante de la matriz de correlaciones.

La validez de criterio se analizó mediante la correlación del cuestionario con dos variables consideradas en la literatura, criterios de referencia, criterios predictivos externos o "gold Standard": "satisfacción global con la atención recibidas en consultas externas" y "conoce el nombre del médico que le atiende en consulta", utilizando el test no paramétrico de correlación de Spearman con cálculo del coeficiente de correlación e intervalo de confianza $95 \%$ en el primer criterio de referencia y la regresión logística, con cálculo de la Odds Ratio e intervalo de confianza 95\% para el segundo.
Se realizó un análisis discriminante ${ }^{26}$, para estimar el porcentaje de pacientes clasificados correctamente mediante el cuestionario en grupos previamente determinados a partir del criterio externo de referencia "Satisfacción global con la atención recibida en consultas externas".

Para analizar la fiabilidad se realizó una evaluación de la consistencia interna mediante el coeficiente Alfa de Cronbach ${ }^{27}$, para el total del cuestionario y para cada uno de los factores de forma independiente, calculándose también la correlación ítemtotal corregido y el valor alfa de Cronbach si el ítem es eliminado.

El análisis estadístico fue realizado con el programa SPSS 14.0.

\section{RESULTADOS}

De las 6.000 encuestas distribuidas entre los pacientes que acudieron a las consultas externas durante el periodo de estudio se recogieron 2.672 , obteniéndose una tasa de respuesta de 44,54\%. 802 encuestas (30\%) fueron desestimadas debido a que no estaban todos los ítems contestados. Sobre las 1.870 encuestas restantes se realizó una selección aleatoria hasta completar el tamaño muestral estimado para el análisis (677 encuestas).

50 pacientes $(7,4 \%)$ acudían a las consultas externas del hospital por primera vez y $594(87,7 \%)$ habían acudido en ocasiones anteriores $(33(4,9 \%)$ no sabe/ no contesta). La edad media de los pacientes incluidos en el análisis fue de 50 años (desviación típica: 18 años). La tabla 1 presenta las características sociodemográficas de los pacientes.

El análisis factorial identificó una estructura en dos factores (medida de adecuación muestral de Kaiser-Meyer-Olkin 0,91, test de esfericidad de Bartlett $\mathrm{p}<0,005$, determinante de la matriz de correlaciones $<0,001$ ) 
Tabla 1

Características sociodemográficas de los pacientes encuestados

\begin{tabular}{|c|c|}
\hline Sexo & $\mathbf{N}(\%)$ \\
\hline Varón & $267(39,4 \%)$ \\
\hline Mujer & $402(59,4 \%)$ \\
\hline No sabe/ no contesta & $8(1,2 \%)$ \\
\hline Nivel de estudios & $\mathbf{N}(\%)$ \\
\hline Sin estudios & $56(8,3 \%)$ \\
\hline Estudios primarios & $246(36,3 \%)$ \\
\hline Estudios secundarios & $191(28,2)$ \\
\hline Universitarios & $162(23,9 \%)$ \\
\hline No sabe/ no contesta & $22(3,2 \%)$ \\
\hline Situación laboral & $\mathbf{N}(\%)$ \\
\hline Empleado & $285(42,1 \%)$ \\
\hline Parado & $52(7,7 \%)$ \\
\hline Pensionista & $188(27,8 \%)$ \\
\hline Estudiante & $30(4,4 \%)$ \\
\hline Ama de casa & $99(14,6 \%)$ \\
\hline No sabe/ no contesta & $23(3,4 \%)$ \\
\hline Nacionalidad & $\mathbf{N}(\%)$ \\
\hline Española & $653(96,5 \%)$ \\
\hline Otra & $17(2,5 \%)$ \\
\hline No sabe/ no contesta & $7(1,0 \%)$ \\
\hline
\end{tabular}

que explicaba el $63 \%$ de la varianza. El primer factor, denominado "Calidad clínica", contenía 6 ítems y explicaba el 36\% de la varianza. El segundo factor, denominado "Calidad administrativa", incluía 6 cuestiones y explicaba el $27 \%$ de la varianza. La tabla 2 muestra los resultados del análisis factorial.
La validez de criterio concurrente respecto al criterio de referencia "satisfacción global con la atención recibidas en consultas externas" obtuvo un coeficiente de correlación de Spearman de 0,85 (IC 95\% 0,83-0,87) para el factor "Calidad Clínica" y 0,69 (IC 95\% 0,64-0,72) para el factor "Calidad Administrativa". La validez concurrente respecto al criterio "conoce del nombre del médico que le atiende en la consulta", dio como resultado una Odds Ratio de 1,19 (IC95\% 1,07-1,32).

Se realizó un análisis discriminante respecto al criterio predicitivo externo "Satisfacción global con la atención recibida en consultas externas", obteniéndose que el $84 \%$ de los casos había sido correctamente clasificado.

Para evaluar la consistencia interna se obtuvo un valor alfa de Cronbach para el cuestionario de 0,90. Los valores de correlación ítem-total oscilaron entre 0,52 y 0,73 . Todas las preguntas fueron contestadas por más del $99 \%$ de los pacientes. El valor alfa de Cronbach para el factor Calidad clínica fue de 0,91 ( $\mathrm{N}^{\mathrm{o}}$ casos: 644), oscilando los valores de las correlaciones ítem-total entre 0,61 y 0,84 . El valor Alfa de Cronbach para el factor Calidad administrativa fue de 0,83 ( $\mathrm{N}^{\mathrm{o}}$ casos: 663 ) con unos valores de las correlaciones ítem-total que

Tabla 2

Resultados del análisis factorial exploratorio y saturación factorial de los ítems tras rotación Varimax

\begin{tabular}{|l|c|c|c|}
\hline \multicolumn{1}{|c|}{ Preguntas del cuestionario } & Factor 1 & Factor 2 & Comunalidades \\
\hline Demora cita & & 0,680 & 0,524 \\
\hline Señalización de las consultas & & 0,680 & 0,498 \\
\hline Facilidad trámites de admisión & & 0,763 & 0,636 \\
\hline Tiempo de espera en consulta & & 0,713 & 0,557 \\
\hline Comodidad de la sala de espera & & 0,729 & 0,554 \\
\hline Trato personal enfermería & 0,610 & 0,376 & 0,514 \\
\hline Trato personal médico & 0,848 & & 0,762 \\
\hline Intimidad de la consulta & 0,794 & & 0,682 \\
\hline Duración de la consulta & 0,774 & & 0,674 \\
\hline Información que da el médico & 0,858 & & 0,787 \\
\hline Claridad de la información & 0,882 & & 0,816 \\
\hline Facilidad trámites para recita & 0,520 & & 0,572 \\
\hline
\end{tabular}


Tabla 3

Correlación ítem-total para cada variable del factor Calidad clínica Calidad administrativa y valor Alfa de Cronbach tras la exclusión de la variable

\begin{tabular}{|c|c|c|}
\hline & Correlación ítem-total & Alfa tras exclusión ítem \\
\hline \multicolumn{3}{|c|}{ Calidad clínica (Alfa de Cronbach 0,91) } \\
\hline Claridad de la información & 0,84 & 0,89 \\
\hline Información que da el médico & 0,82 & 0,89 \\
\hline Trato personal médico & 0,82 & 0,89 \\
\hline Intimidad de la consulta & 0,75 & 0,90 \\
\hline Duración de la consulta & 0,73 & 0,90 \\
\hline Trato personal enfermería & 0,61 & 0,92 \\
\hline \multicolumn{3}{|c|}{ Calidad administrativa (Alfa de Cronbach 0,83) } \\
\hline Facilidad trámites de admisión & 0,66 & 0,79 \\
\hline Comodidad de la sala de espera & 0,57 & 0,80 \\
\hline Tiempo de espera en consulta & 0,60 & 0,80 \\
\hline Demora cita & 0,60 & 0,80 \\
\hline Señalización de las consultas & 0,55 & 0,81 \\
\hline Facilidad trámites para recita & 0,60 & 0,80 \\
\hline
\end{tabular}

Anexo 1

Cuestionario de Satisfacción del Usuario de consultas externas (SUCE)

\begin{tabular}{|l|l|l|l|l|l|l|l|l|l|l|l|}
\hline \multicolumn{7}{|c|}{ Por favor puntúe de 1 a 10 las siguientes preguntas, siendo el 10 la respuesta mejor valorada y el 1 la respuesta peor valorada } \\
\hline 1. El tiempo que pasó desde que pidió la cita hasta la fecha de consulta & 1 & 2 & 3 & 4 & 5 & 6 & 7 & 8 & 9 & 10 \\
\hline 2. Las señalizaciones y carteles para orientarse en el hospital & 1 & 2 & 3 & 4 & 5 & 6 & 7 & 8 & 9 & 10 \\
\hline 3. Los trámites que tuvo que hacer en Admisión & 1 & 2 & 3 & 4 & 5 & 6 & 7 & 8 & 9 & 10 \\
\hline 4. El tiempo de espera en consultas & 1 & 2 & 3 & 4 & 5 & 6 & 7 & 8 & 9 & 10 \\
\hline 5. La comodidad de la sala de espera & 1 & 2 & 3 & 4 & 5 & 6 & 7 & 8 & 9 & 10 \\
\hline 6. El trato por parte del personal de enfermería & 1 & 2 & 3 & 4 & 5 & 6 & 7 & 8 & 9 & 10 \\
\hline 7. El trato por parte del personal médico & 1 & 2 & 3 & 4 & 5 & 6 & 7 & 8 & 9 & 10 \\
\hline 8. El cuidado con su intimidad durante la consulta & 1 & 2 & 3 & 4 & 5 & 6 & 7 & 8 & 9 & 10 \\
\hline 9. La duración de la consulta & 1 & 2 & 3 & 4 & 5 & 6 & 7 & 8 & 9 & 10 \\
\hline 10. La información clínica recibida sobre su problema de salud & 1 & 2 & 3 & 4 & 5 & 6 & 7 & 8 & 9 & 10 \\
\hline 11. La claridad con que le explicaron el tratamiento y pautas & 1 & 2 & 3 & 4 & 5 & 6 & 7 & 8 & 9 & 10 \\
\hline 12. La facilidad de los trámites que ha tenido que hacer si ha necesitado volver & 1 & 2 & 3 & 4 & 5 & 6 & 7 & 8 & 9 & 10 \\
\hline a citarse & & & & & & & \\
\hline
\end{tabular}

oscilaron entre 0,55 y 0,66 . La tabla 3 muestra el valor de la correlación ítem-total para cada variable y el valor alfa de Cronbach cuando el ítem es excluido.

\section{Versión final del cuestionario}

Como resultado del proceso de validación se obtuvo un cuestionario final (anexo 1) compuesto por 12 ítems que determinan dos factores. Cada uno de los ítems puntúa en una escala desde 1 hasta 10. La puntua- ción final del cuestionario se obtiene como la media de la puntuación media de las dos dimensiones.

\section{DISCUSIÓN}

En este trabajo se presenta un cuestionario que ha sido diseñado específicamente para conocer la satisfacción de los pacientes con las consultas externas hospitalarias. El cuestionario presenta la ventaja de un número reducido de ítems, es fácilmente 
comprensible por los pacientes y se puede responder en un tiempo breve. La utilización de una escala de respuesta de 1 a 10 resulta sencilla e intuitiva para el paciente, si bien puede favorecer la tendencia a contestar alrededor de los valores centrales.

El cuestionario SUCE permite obtener una medida de la satisfacción que engloba todo el proceso asistencial y no asistencial de las consultas, debido a que abarca todos los aspectos de la atención, desde que el paciente solicita la citación para la consulta hasta que acude y es atendido en la misma. Además, se puede aplicar a los pacientes que acuden a cualquiera de las consultas externas hospitalarias, independientemente de la especialidad.

El hecho de que el cuestionario presente una primera parte para contestar antes de entrar en la consulta y una segunda para contestar a la salida de la misma, permite que los pacientes puedan ir leyendo el cuestionario y respondiendo mientras están esperando, antes de entrar en la consulta, lo que aumenta la tasa de respuesta. Sin embargo, la desventaja que hemos encontrado es que hay pacientes que responde a la primera parte del cuestionario pero no a la segunda, lo que puede ser debido al "olvido", "prisa por abandonar las consultas", etc.

El cuestionario SUCE ha demostrado tener una buena estructura factorial, una buena capacidad predictiva y una alta consistencia interna. El análisis factorial exploratorio de componentes principales identificó una estructura en dos factores que explicaba el $63 \%$ de la varianza. La denominación de los factores se realizó intentando encontrar un nexo común que englobara las distintas variables incluidas en cada uno de ellos. El primer factor, denominado "Calidad Clínica", incluye aspectos relacionados propiamente con la atención recibida durante la consulta desde el personal sanitario, como son el trato y la información y la protección de la intimidad, así como la calidad científico-técnica. En este factor, se englobaban por tanto, variables más dependientes del personal sanitario y del propio acto médico. El segundo factor, denominado "Calidad Administrativa" incluía aspectos organizativos y estructurales como la comodidad de las instalaciones, la facilidad de los trámites o los tiempos de espera. En este factor se reunían aspectos de tipo burocrático más relacionados con la gestión. Esta estructura en dos factores parece adecuada por representar un equilibrio entre explicar el máximo porcentaje de varianza y utilizar un modelo lo más parsimonioso posible. Las saturaciones factoriales que indican la influencia de cada variable en el factor son elevadas, de forma que cada variable explica gran parte de la información aportada por el factor.

El análisis de la validez de criterio concurrente respecto a los criterios de referencia "satisfacción global con la atención recibida en consultas externas" y "conoce el nombre del médico que le atiende en consulta" indicó una óptima validez de criterio del cuestionario, especialmente en lo que respecta al factor "calidad clínica" y una suficiente interrelación con una medida global de la satisfacción del paciente.

Mediante análisis discriminante se confirmó que el porcentaje de casos clasificados correctamente en virtud de las puntuaciones del cuestionario SUCE fue del 84\% sobre el criterio "Satisfacción global con la atención recibida en consultas externas", lo que supone una óptima capacidad predictiva del cuestionario, siendo adecuado para discriminar entre pacientes satisfechos e insatisfechos. La fiabilidad del cuestionario medida mediante el valor Alfa de Cronbach aportó un valor superior a 0,80 en todos los casos, no mejorables tras la exclusión de ninguna de las variables, indicando que el cuestionario presenta una elevada consistencia interna. 
Según los resultados obtenidos, podemos afirmar que el cuestionario elaborado para medir la satisfacción de los pacientes en consultas externas es una herramienta útil y confiable, por lo que podemos recomendar su aplicación. No obstante, como en cualquier otro instrumento de estas características, es necesario que su utilización se haga en condiciones semejante a aquellas en que ha sido validado, ya que su utilización en otro contexto como Centro de Especialidades Periféricas o Centros de Atención Primaria puede requerir una adaptación. Por otra parte, consideramos necesario administrar este cuestionario en sucesivas ocasiones con objeto de valorar la respuesta al cambio.

\section{AGRADECIMIENTOS}

Los autores agradecen la colaboración prestada para la realización de este trabajo a Amparo Mancebo Izco, Jefa de Área de Estudios Sociológicos de la Dirección General de Calidad de los Servicios y Atención al Ciudadano de la Comunidad de Madrid, a José María Bellón Cano, estadístico del Servicio de Medicina Preventiva y Gestión de Calidad del Hospital General Universitario Gregorio Marañón y al equipo de administrativos del Servicio de Medicina Preventiva y Gestión de Calidad por su colaboración en el procesamiento de datos.

\section{BIBLIOGRAFÍA}

1. AENOR. Guía para la implantación de sistemas de calidad ISO 9000 en la PYME de comercio y servicios. Madrid: AENOR; 2000.

2. Aguirre, H. Evaluación de la calidad de la atención médica. Expectativas de los pacientes y de los trabajadores de las unidades médicas. Salud Publica Mex. 1990; 32:170-80.

3. Martínez Ramírez A, Van-Dick Puga MA, Nápoles Rodríguez F, Robles Uribe J, Ramos Ramos A, Villasenor Urrea I. Towards a strategy for quality assurance: satisfaction in the utilization of medical care. Cad Saude Publica. 1996; 12:399-403.

4. Zastowny TR, Roghman KJ, Cafferata GL. Patient satisfaction and the use of health services. Med Care. 1989; 27:705-23.

5. Prieto Rodríguez MA, March Cerdá JC, López Fernández LA. Calidad percibida por usuarios de centros de salud y de aseguradoras privadas. Aten primaria. 1999; 24:259-66.

6. Moliner- Tena MA, Moliner-Tena J. La calidad percibida de un servicio de un centro de planificación familiar: un enfoque de marketing. Aten Primaria. 1996; 17:400-6.

7. Donabedian A. Evaluating the quality of medical care. Milbank Memorial Fund Quart. 1966; 44:166-202.

8. Lewis JR. Patient views on quality care in general practice: literature review. Soc Sci Med. 1994; 39:655-70.

9. Pascual L, Uris J, Alfonso MD, Sanmartín D, Sanz J, Campos C. Las reclamaciones y las hojas de sugerencias como método de incorporación de la opinión del usuario en atención primaria. Comparación con la perspectiva de los profesionales. Aten Primaria. 1995; 15:421-5.

10. Saturno PJ. Los métodos de participación del usuario en la evaluación y mejora de la calidad de los servicios sanitarios. Rev Esp Salud Pública. 1995; 69:163-75.

11. Crow R, Gage H, Hampson S et al. The measurement of satisfaction with healthcare: implications for practise from a systematic review of the literature. Health Technol Assess. 2002; 6:1-245.

12. Hail, JA, Dorman MC. A meta-analysis of satisfaction with medical care: Description of research domain and analysis of overall satisfaction levels. Soc Sci Med. 1998; 27:637-44.

13. Bretones C, Sánchez JE. Construcción y validación de una encuesta de satisfacción de usuarios de atención primaria. Aten Primaria. 1989; 6:312-6.

14. McKinley RK, Manku-Scott T, Hastings AM, French DP, Baker R. Reliability and validity of a new measure of patient satisfaction with out of hours primary medical care in the United Kingdom: development of a patient questionnaire. BMJ. 1997; 314:193-8.

15. Labarere J, Francois P, Auquier P, Robert C, Fourny M. Development of a French impatient 
satisfaction questionnaire. Int J Qual Health Care. 2001; 13:99-108.

16. Pérez Blanco V, García Caballero J, Cisneros J, Tabernero A. Satisfacción del paciente urológico en consultas externas. Rev Calidad Asistencial. 2003; 18:628-35.

17. Ramírez-Hernández M, Mérida Fernández C, Negro Álvarez JM, Félix Toledo R, Martínez López RM. Satisfacción de los pacientes asistidos en una consulta externa de alergología. Alergol Inmunol Clin. 2004; 19: 145-52.

18. Boix JH, Álvarez F, Tejeda M, Sáiz E. Análisis de opinión de los usuarios sobre la asistencia prestada en las consultas externas de un hospital y su centro de especialidades: componentes y diferencias. Gest Hosp. 1994; 4:32-8.

19. Conesa A, Bayas JM, Asenjo MA, Bare ML, Manasanch o, Lledó R et al. Calidad percibida por los usuarios de consultas externas de un hospital universitario. Rev Clin Esp. 1993; 192:346-51.

20. Barrasa JI, Aibar C. Revisión sistemática de los estudios de satisfacción realizados en España en el periodo 1986-2001. Rev Calidad Asistencial. 2003; 18: 580- 90 .

21. Anden A, Andersson SO, Rudebeck CE. Satisfaction is not all patients' perceptions of outcome of general practice consultations, a qualitative study. BMC Fam Pract. 2005; 6: 43.

22. Gasquet I, Villeminot S, Estaquio C, Duriex P, Ravaud P, Falissard B. Construction of a questionnaire measuring outpatients'opinion of hospital consultation departments. Health Qual Life Outcomes. 2004; 2:43.
23. Maderuelo JA, Carriedo E, Serrano M, Almaraz A, Berjón AC. Factores de insatisfacción con el sistema sanitario. Un estudio Delphi. Aten Primaria. $1996 ; 31: 348-52$.

24. Thompson AG, Suñol R. Expectations as determinants of patient satisfaction: concepts theory and evidence. Int J Qual Health Care. 1995; 7:127-41.

25. Díaz de Rada Igurquiza V. Análisis Factorial. En: Díaz de Rada Igurquiza V. Técnicas de análisis multivariante para investigación social y comercial. Madrid: RA-MA; 2002. p. 91-156.

26. Salvador Figueras M. Análisis Discriminante [citado 14 de may. 2007]. Disponible en: www.5campus.com/leccion/discri

27. Cronbach LJ. Coefficient alpha and the internal structure of test. Psychometrika. 1951; 16: 297 334.

28. Linder-Pelz SU. Toward a theory of patient satisfaction. Soc Sci Med. 1982; 16:577-82.

29. Linder-Pelz S. Social psychological determinants of patient satisfaction: a test of five hypothesis. Soc Sci Med. 1982; 16:583-89.

30. McKinley RK, Manku-Scott T, Hastings AM, French DP, Baker R. Reliability and validity of a new measure of patient satisfaction with out of hours primary medical care in the United Kingdom: development of a patient questionnaire. BMJ. 1997; 314:193-8.

31. González L, Costa A, Timoneda C, Alfonso JL y Cortina P. Encuesta de satisfacción a los usuarios de un centro de salud. Gac Sanit. 1993; 7:86-94. 
